\title{
Impact of nuclear structure on the production and identification of superheavy nuclei
}

\author{
N.V. Antonenko", a, G.G. Adamian ${ }^{1}$, A.N. Bezbakh ${ }^{1}$, and T.M. Shneidman ${ }^{1}$ \\ ${ }^{1}$ Joint Institute for Nuclear Research, 141980 Dubna, Russia
}

\begin{abstract}
The shell structure of heavy nuclei with $Z>104$, which can be produced in actinide-based complete fusion reactions, is studied with a modified two-center shell model. Using the macroscopic-microscopic approach, mass excesses and $Q_{\alpha}$-values are calculated and compared with available experimental data. The production cross sections of new superheavy nuclei decisively depend on the position of the proton shell closure.
\end{abstract}

\section{Introduction}

The experiments on complete fusion reactions with ${ }^{48} \mathrm{Ca}$ beam and various actinide targets were successfully carried out at FLNR (Dubna), GSI (Darmstadt), and LBNL (Berkeley) [1-6] in order to synthesize superheavy nuclei with $Z=112-118$. The found experimental trend of the nuclear properties ( $Q_{\alpha}$-values and half-lives) and cross sections of the superheavy elements (SHE) produced with ${ }^{48} \mathrm{Ca}$-induced reactions reveals the increasing stability of nuclei approaching the spherical closed neutron shell $N=184$, and also indicates a relatively small effect of the proton shell at $Z=114[7,8]$. With the microscopic-macroscopic models [9-12] "the island of stability" of the SHE is predicted at charge number $Z=114$ and neutron number $N=184$. In accordance with predictions of the relativistic and nonrelativistic mean field models [13-15], the most stable nuclei have $Z=120-126$ and $N=184$. If this is true, then there is a hope to synthesize new SHE with $Z \geq 119$ by using the present experimental set-ups and actinide-based reactions with neutron-rich stable projectiles heavier than ${ }^{48} \mathrm{Ca}$.

\section{Modified microscopic-macroscopic approach}

The stability of superheavies is related to the shell effects which are ruled by the mean field and the spin-orbit interaction. In Ref. [16] we proposed a microscopic-macroscopic approach based on the two-center shell model (TCSM) [17]. The parameters were set so to describe in the best way the spins and parities of the ground state of heavy nuclei. With this modified microscopic-macroscopic approach one can reveal the trends in the shell effects and $Q_{\alpha}$ values with $Z$. Note that the global fit is out of our task and the obtained binding energies are the subject of further improvement.

Besides our results, the microscopic-macroscopic models [9-12] as well as the phenomenological model [18] provide us the $Q$ values of the reactions, fission barriers and neutron separation energies of

\footnotetext{
a e-mail: antonenk@theor.jinr.ru
} 
superheavy nuclei which are necessary to calculate evaporation residue cross sections $\sigma_{E R}$. The value of survival probability strongly depends on $B_{f}-B_{n}$, the difference between the height $B_{f}$ of the fission barrier and the neutron separation energy $B_{n}$. The values of $B_{n}$ predicted with different models vary within $0.5 \mathrm{MeV}$ and the shell effects or $B_{f}$ cause the main difference in the dependencies of $B_{f}-B_{n}$ on $N$ [19] in comparison with the predictions of Refs. [9, 18]. As follows, for the compound nuclei with $Z=120-124$ we expect larger survival probabilities than for the nuclei with $Z=114$.

As seen in Fig. 1, the calculated $Q_{\alpha}$ are in a good, within $0.3 \mathrm{MeV}$, agreement with the available experimental data. The shell at $N=162$ is less pronounced in our calculations than in Refs. [9-12] The shell effects at $Z=114$ and $N=172-176$ provide rather weak dependence of $Q_{\alpha}$ on $N$. The strong role of the shell at $Z=120$ and $N=184$ is reflected in the well pronounced minimum of $Q_{\alpha}$. As in our calculations, there is strong evidence of the shell closure at $N=184$ in the phenomenological model [18].

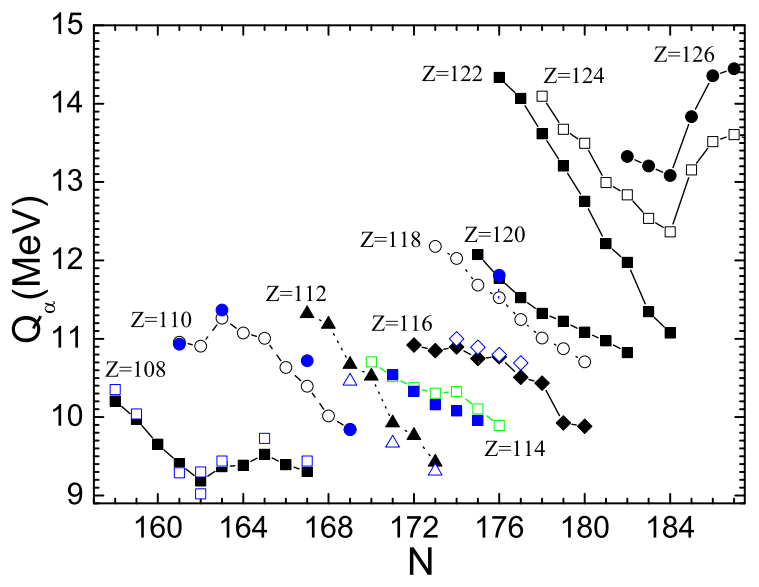

Figure 1. Calculated $\alpha$-decay energies (symblos connected by lines) are compared with available experimental data (symbols) $[1,2,5]$ for even- $Z$ nuclei with $Z \geq 108$.

To shed light on the dependence of the level density on the shell effects, the calculated energy dependencies of the level densities [20] are fitted by the well-known expression of the Fermi-gas model. Then we obtain the dependence of the level density parameter $a$ on $Z, N$, and excitation energy. We consider the dependencies of $a$ on $Z$ (Fig. 2) for three $\alpha$-decay chains containing the nuclei ${ }^{296,298,300} 120$ which could be synthesized with available projectiles and targets. At $Z=108$ and 120 there are minima of $a$ in all chains. This reflects quite a strong proton shell effects at $Z=108$ and 120 . At $Z=120$, the minima of $a$ are the deepest and well pronounced. The similar behavior of $a$ occurs near $Z=82$. As in Ref. [19], we conclude here that the modified TCSM provides the proton shell closure at $Z=120$. The sub-shell at $Z=114$ exists but provides weaker shell effect than at $Z=120$. For nuclei with $Z=124-128$, the minima of $a$ are due to the neutron shell at $N=184$.

The dinuclear system model [21-25] is successful in describing fusion-evaporation reactions especially related to the production of superheavy nuclei. Using our predictions of nuclear properties [19], we calculated the values of $\sigma_{E R}$ in the reactions ${ }^{48} \mathrm{Ca},{ }^{50} \mathrm{Ti},{ }^{54} \mathrm{Cr},{ }^{58} \mathrm{Fe},{ }^{64} \mathrm{Ni}+{ }^{238} \mathrm{U},{ }^{244} \mathrm{Pu},{ }^{248} \mathrm{Cm},{ }^{249} \mathrm{Cf}$ (Fig. 3). In comparison to our previous calculations with the mass table of Ref. [9], in Fig. 3 the values of $\sigma_{E R}$ decreases slower with increasing $Z$. The stronger shell effects revealed here for nuclei with $Z>118$ result in larger survival probabilities and 
INPC 2013

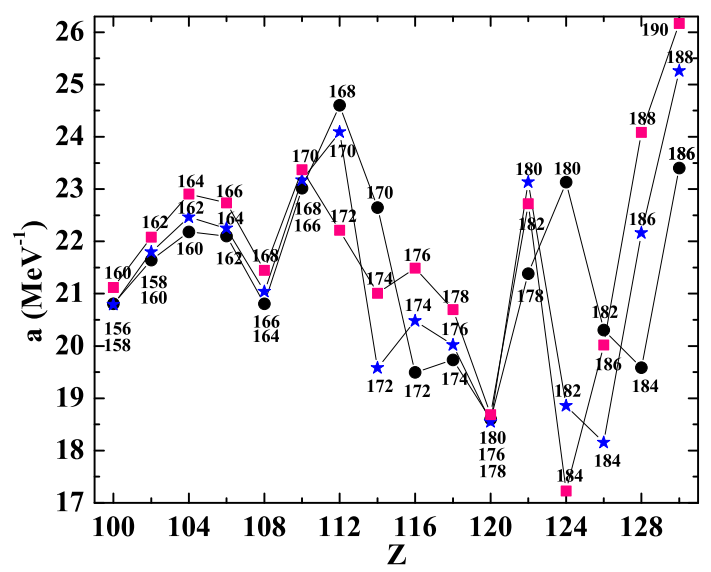

Figure 2. Calculated parameter of level density as a function of $Z$ for nuclei of alpha-decay chains containing $296,298,300120$. Neutron numbers are given at the corresponding data points.

larger values of $\sigma_{E R}$. A good description of existing data allows us to be confident in the predictions for the reactions with heavier projectiles.

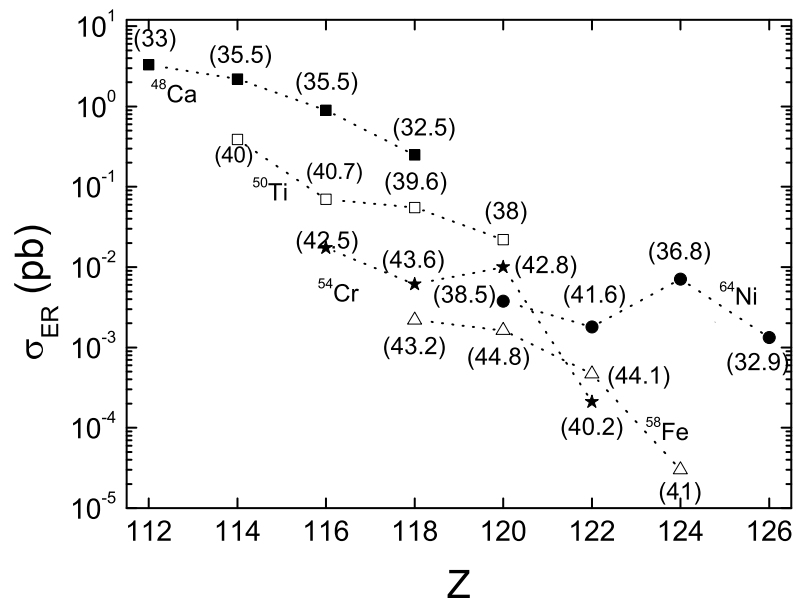

Figure 3. The evaporation residue cross sections in the maxima of excitation functions versus charge number $Z$ for the reactions ${ }^{48} \mathrm{Ca},{ }^{50} \mathrm{Ti},{ }^{54} \mathrm{Cr},{ }^{58} \mathrm{Fe},{ }^{64} \mathrm{Ni}+{ }^{238} \mathrm{U},{ }^{244} \mathrm{Pu},{ }^{248} \mathrm{Cm},{ }^{249} \mathrm{Cf}$. The excitation energies of compound nuclei are given in brackets.

With ${ }^{50} \mathrm{Ti}$ beam the values of $\sigma_{E R}$ for the nuclei with $Z=114-118$ are expected to be $5-10$ times smaller than those resulting for ${ }^{48} \mathrm{Ca}$ beam. The main reason for this is the decrease of fusion probability with mass asymmetry in the entrance channel of reaction. With ${ }^{50} \mathrm{Ti}$ the nucleus ${ }^{295} 120$ is predicted to be produced with the cross section of $23 \mathrm{fb}$. In the ${ }^{54} \mathrm{Cr}+{ }^{248} \mathrm{Cm}$ reaction the compound nucleus would have 3 neutrons more than in the ${ }^{50} \mathrm{Ti}+{ }^{249} \mathrm{Cf}$ reaction. Therefore, the decrease of fusion probability is partly compensated by the increase of survival probability and the nucleus ${ }^{298} 120$ could 
be produced with the cross section of $10 \mathrm{fb}$. For the production of nuclei with $Z=122-126,{ }^{64} \mathrm{Ni}$ beam would lead to larger cross sections, $1-8 \mathrm{fb}$.

\section{Summary}

The calculations performed with the modified TCSM reveal quite strong shell effects at $Z=120-126$ and $N=184$ as in the self-consistent mean-field treatments. If our prediction of the structure of heaviest nuclei is correct, than one can expect the production of evaporation residues $Z=120$ in the reactions ${ }^{50} \mathrm{Ti}+{ }^{249} \mathrm{Cf}$ and ${ }^{54} \mathrm{Cr}+{ }^{248} \mathrm{Cm}$ with the cross sections 23 and $10 \mathrm{fb}$, respectively. The $Z=120$ nuclei with $N=175-179$ are expected to have $Q_{\alpha}$ about $12.1-11.2 \mathrm{MeV}$ and lifetimes $1.7 \mathrm{~ms}-0.16$ $\mathrm{s}$ in accordance with our predictions. The experimental measurement of $Q_{\alpha}$ for at least one isotope of $Z=120$ would help us to set proper shell gaps in the region of SHE.

This work was supported in part by RFBR. The IN2P3 (France)-JINR (Dubna), and Polish - JINR (Dubna) Cooperation Programmes are gratefully acknowledged.

\section{References}

[1] Yu.Ts. Oganessian, J. Phys. G 34, R165 (2007)

[2] Yu.Ts. Oganessian et al., Phys. Rev. C 87, 054621 (2013)

[3] S. Hofmann et al., Eur. Phys. J. A 48, 62 (2012)

[4] S. Heinz et al., J. Phys.: Conf. Ser. 282, 012007 (2011)

[5] L. Stavsetra, K.E. Gregorich, J. Dvorak, P.A. Ellison, I. Dragojević, M.A. Garcia, and H. Nitsche, Phys. Rev. Lett. 103, 132502 (2009)

[6] Ch.E. Düllmann et al., Phys. Rev. Lett. 104, 252701 (2010)

[7] G.G. Adamian, N.V. Antonenko, and V.V. Sargsyan, Phys. Rev. C 79, 054608 (2009)

[8] G.G. Adamian, N.V. Antonenko, V.V. Sargsyan, and W. Scheid, Nucl. Phys. A834, 345c (2010)

[9] P. Möller, J.R. Nix, W.D. Myers, and W.J. Swiatecki, At. Data Nucl. Data Tables 59, 185 (1995)

[10] I. Muntian, Z. Patyk, and A. Sobiczewski, Acta. Phys. Pol. B 32, 691 (2001)

[11] I. Muntian, Z. Patyk, and A. Sobiczewski, Acta. Phys. Pol. B 34, 2141 (2003)

[12] A. Parkhomenko and A. Sobiczewski, Acta. Phys. Pol. B 36, 3095 (2005)

[13] P. Ring, Prog. Part. Nucl. Phys. 37, 193 (1996)

[14] M. Bender, P.H. Heenen, and P.G. Reinhard, Rev. Mod. Phys. 75, 121 (2003)

[15] J. Meng, H. Toki, S.G. Zhou, S.Q. Zhang, W.H. Long, and L.S. Geng, Prog. Part. Nucl. Phys. 57, $470(2006)$

[16] G.G. Adamian, N.V. Antonenko, and W. Scheid, Phys. Rev. C 81, 024320 (2010)

[17] J. Maruhn and W. Greiner, Z. Phys. A 251, 431 (1972)

[18] S. Liran, A. Marinov, and N. Zeldes, Phys. Rev. C 62, 047301 (2000)

[19] A.N. Kuzmina, G.G. Adamian, N.V. Antonenko, and W. Scheid, Phys. Rev. C 85, 014319 (2012)

[20] A.N. Bezbakh et al., to be published

[21] N.V. Antonenko, E.A. Cherepanov, A.K. Nasirov, V.P. Permjakov, and V.V. Volkov, Phys. Lett.

B 319, 425 (1993)

[22] G.G. Adamian, N.V. Antonenko, W. Scheid and V.V. Volkov, Nucl. Phys. A 633, 409 (1998)

[23] G.G. Adamian, N.V. Antonenko and W. Scheid, Nucl. Phys. A 678, 24 (2000)

[24] G.G. Adamian, N.V. Antonenko, and W. Scheid, Phys. Rev. C 69, 014607 (2004)

[25] G.G. Adamian, N.V. Antonenko, and W. Scheid, Lecture Notes in Physics, Clusters in Nuclei 848, ed. by C. Beck (Springer, Berlin, 2012) p. 165-227 